\title{
Robotic removal of Müllerian duct remnants in pediatric patients: our experience and a review of the literature
}

\author{
Mario Lima, ${ }^{1}$ Michela Maffi, ${ }^{1}$ Niel Di Salvo, ${ }^{1}$ Giovanni Ruggeri, ${ }^{1}$ Michele Libri, ${ }^{1}$ Tommaso Gargano, ${ }^{1}$ \\ Hubert Lardy 2
}

1Sant'Orsola Hospital, Department of Pediatric Surgery, University of Bologna, Bologna, Italy; ${ }^{3}$ Tours University-Hospital Centre, Tours, France

\begin{abstract}
Persistent Müllerian duct syndrome is a disorder of sexual development, which features a failure of involution of Müllerian structures. An enlarged prostatic utricle is a kind of Müllerian duct remnant (MDR) with a tubular shaped structure communicating with the prostatic urethra. Treatment is aimed at relieving symptoms when present, preserve fertility and prevent neoplastic degeneration. We describe 3 cases of successful robot assisted-removal of symptomatic MDRs. The first case came to our attention for pseudo-incontinence; the other two for recurrent urinary tract infections. The patients have not presented such symptoms anymore on follow-up. We then reviewed existent literature on authors who have recently investigated the main issues concerning MDRs and have attempted a roboticassisted approach on them. Robot-assisted laparoscopy can be considered a valid, safe and effective minimally-invasive technique for the primary treatment of prostatic utricle.
\end{abstract}

\section{Introduction}

Persistent Müllerian duct syndrome (PMDS) is a disorder of sexual development, which features a failure of involution of

\footnotetext{
Correspondence: Mario Lima, Sant'Orsola Hospital, Department of Pediatric Surgery, University of Bologna, Via Massarenti 11, 40138 Bologna, Italy.

Tel.: +39.333.58130225.

E-mail:mario.lima@unibo.it

Key words: Müllerian duct remnants; Robotic surgery; Enlarged prostatic utricle.

Conflict of interest: the authors declare no potential conflict of interest.

Funding: none.

Received for publication: 16 December 2017.

Accepted for publication: 22 May 2018.

This work is licensed under a Creative Commons Attribution NonCommercial 4.0 License (CC BY-NC 4.0).

CCopyright M. Lima et al., 2018

Licensee PAGEPress, Italy

La Pediatria Medica e Chirurgica 2018; 40:182

doi:10.4081/pmc.2018.182
}

Müllerian structures including a uterus, a cervix, fallopian tubes and the upper two thirds of vagina. Their persistency could be influenced by a hormonal imbalance in anti-Müllerian hormone (AMH) and testosterone secretion or the AMH responsiveness of target tissues during a short critical period at the end of the undifferentiated stage (weeks 9 to 10). An enlarged prostatic utricle (EPU) is a kind of Müllerian duct remnant (MDR) with a tubular shaped structure communicating with the prostatic urethra. Symptoms such as recurrent urogenital tract infections (UTIs), voiding dysfunctions or urinary incontinence are secondary to urine trapping in the diverticulum. Treatment is aimed at relieving symptoms when present, preserve fertility and prevent neoplastic degeneration. Several open surgical approaches and endoscopic techniques have been used, but laparoscopy has become the gold standard treatment in the last two decades. 1,2 We describe 3 cases of successful robotassisted removal of MDRs and review existent literature.

\section{Case Reports}

\section{Case $\mathbf{n}^{\circ} 1$}

A 6-year-old child came to our attention for incontinence. He was born on term, no anomalies were suspected on the routine prenatal ultrasound, nothing relevant was reported in the medical history, external genitalia were normal. The parents described involuntary loss of urine in the minutes following a well-controlled valid micturition. Ultrasound detected a hypo echogenic tubularlike structure located in the retro prostatic space, which was correctly interpreted as an MDR. The latter was then identified as an EPU while performing a voiding cystourethrography (VCU) that showed the connection between the urethra and the structure and excluded other hidden associated uropathies (Figure 1A). This patient also underwent magnetic resonance imaging (MRI) to provide better anatomical definition of the internal genitalia and plan the correct surgical strategy (Figure 1B).

The patient's issue was therefore better defined as pseudoincontinence. We used the DaVinci Xi Robotic Surgical System. The child lies supine with legs stretched out and abducted, enabling concomitant urethrocystoscopy which is performed to place a ureteral catheter inside the utricle and a Foley catheter into the bladder to aid in identification (Figure 2A, B).

The optical port at the umbilicus was advanced into the peritoneum and two 8-mm working ports were placed on the pararectal lines under direct visualization (distance between each port and umbilicus: $7 \mathrm{~cm}$ ). An additional 5-mm assistant port is used. The table is put in Trendelenburg position so that the bowel glides out of the pelvis by gravity; finally the ports are secured to the 
robotic system. After pneumoperitoneum was achieved and MDR dome identified, also through the help of the assistant providing movement of the catheter inside the utricle, the peritoneal reflection covering the dome was incised to free the dome. The MDR was grasped with forceps and carefully dissected free of the surrounding tissues of the retro vesical space with a monopolar hook (Figure $3 \mathrm{~A})$. A complete bloodless dissection was carried out for the entire length of the diverticulum (Figure 3B). Particular attention was given to avoid injury to the bladder neck, urethra, rectum, ureters, vas deferens, prostate and seminal vesicles. In this particular case the vas deferens did not seem to be included within the diverticulum's wall. Once completely dissected, the MDR neck was secured with two preformed endoscopic loops and resected just a few millimetres above its junction with the urethra (Figure 3C, D).

By moving straight and forward the bladder catheter we made sure not to have caused a stricture in the urethra. The structure was excised as much as possible to avoid iatrogenic damage to the urethra such as stenosis but at the same time to avoid residual cyst and recurrence after the procedure. The specimen is removed intact through the camera port. An abdominal drain was put in place and then removed on the fourth post-operative day. The trocar sites were then closed. The Foley catheter was left in place and removed on post-operative day 5. Post-operative course was uneventful and the patient was discharged on day 6 with normal micturition. On a oneyear follow-up, involuntary loss of urine following micturition has not been reported anymore by the patient and his parents.

\section{Case $n^{\circ} 2$}

The case of a 7-year-old boy was investigated for recurrent urinary tract infections. In his medical history, he mainly presented a Denis Drash syndrome; diagnosis was made because of the association between bilateral nephroblastoma and a posterior hypospadias. Surgical management of bilateral nephroblastoma consisted of bilateral nephrectomy at 1 year old and renal transplant. The posterior hypospadias was corrected at 6-years of age and was immediately complicated by a urethral fistula. Investigations regarding recurrent UTIs started after hypospadias correction. A MDR was suspected by ultrasound and confirmed by MRI. The MRI showed a full formation of fluid posterior to the bladder (21

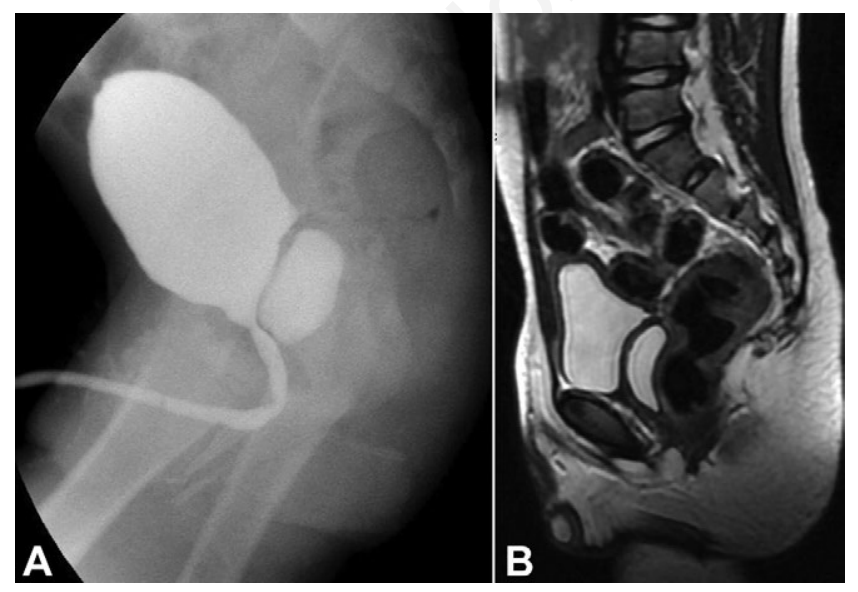

Figure 1. A) Pre-operative voiding cystourethrography showing the connection between the Müllerian duct remnant and the posterior urethra; B) preoperative magnetic resonance imaging providing better anatomical definition of the tubular-like structure in the retrovesical space. $\mathrm{mm}$ in diameter and $44 \mathrm{~mm}$ in height), compatible with An EPU. A cystourethroscopy identified the remnant, with trouble urine inside. We used the DaVinci Xi Robotic Surgical System. The procedure did not differ much from the previous one except for the numerous peritoneal adhesions (due to previous abdominal surgery) that had to be released to allow implementation of the working space. Furthermore, great care was given when the two 8-mm working ports were placed on the left and right flank under direct visualization in order not to damage his transplanted kidney. A complete bloodless dissection was carried out for the entire length
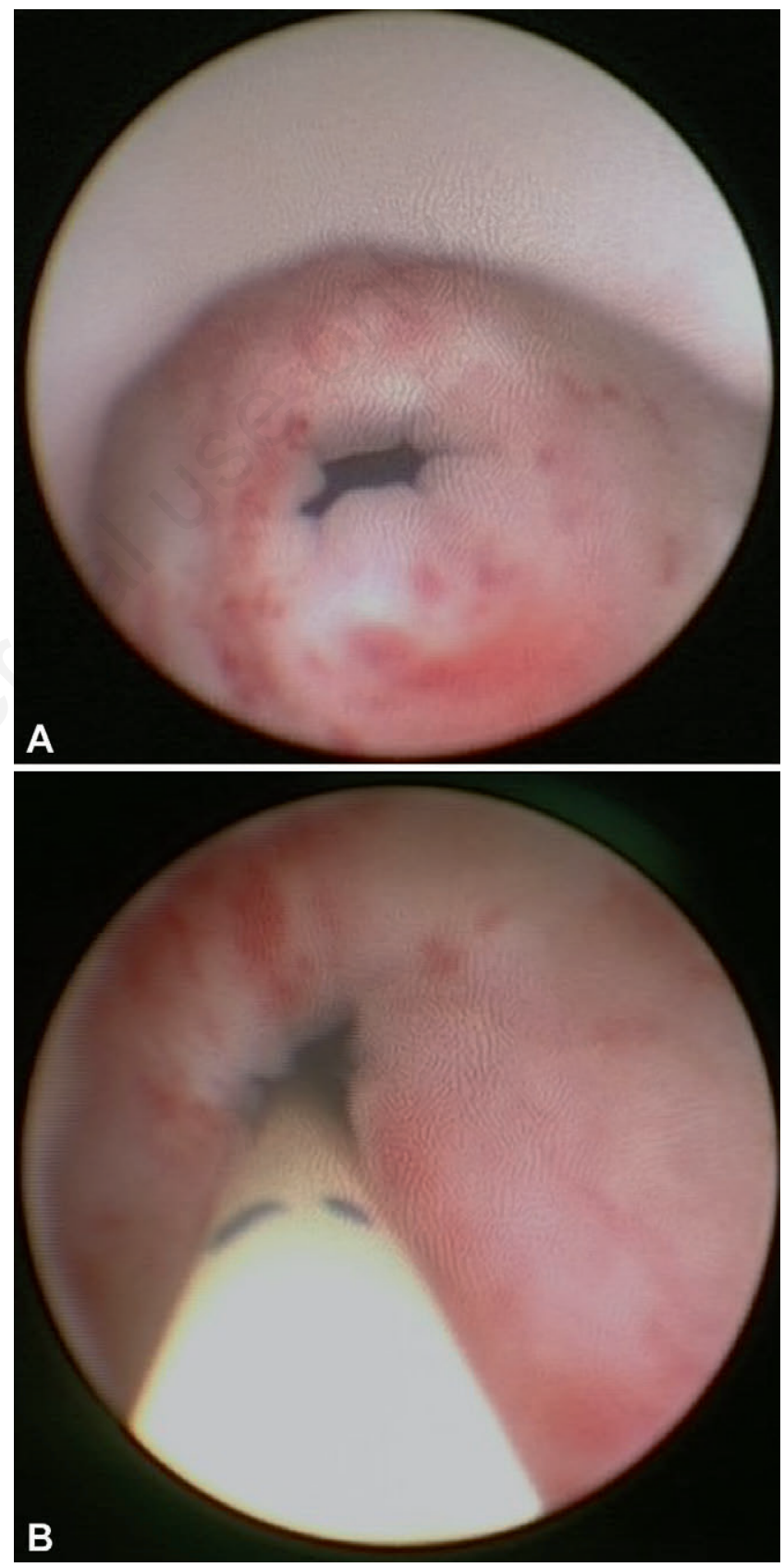

Figure 2. A) At the start of the procedure a cystoscopy was performed showing the entrance of the diverticulum on the posterior urethra; B) during the cystoscopy a ureteral catheter was placed into the Müllerian duct remnant to aid in identification during dissection. 
of the diverticulum. The vas tubes were perfectly identified and respected. The MDR was dissected and cut up to its connection with the posterior urethra. A suture in the section was performed in MAXON ${ }^{\circledR} 5 / 0$. The specimen was removed intact through the camera port. The trocar sites were then closed without leaving any abdominal drain. The Foley catheter was left in place and removed on post-operative day 5. Post-operative course was uneventful and the patient was discharged on day 6 with normal micturition. UTIs did not occur anymore after surgery as reported in a one-year follow-up.

\section{Case $n^{\circ} 3$}

A 3-year-old boy's case had been investigated for recurrent UTIs. An intra-uterine growth restriction secondary to a maternal infection was detected antenatally. At birth, Fanconi's disease was diagnosed; the patient also presented a posterior hypospadias, bilateral undescended testes, a severe bilateral hypoplasia of the thumb and a bilateral clubfoot. According to Fanconi's disease, he presented a horseshoe kidney associated with a vesicoureteral reflux (VUR). A bilateral vesicoureteral re-implantation was performed at 2 years of age. He went on presenting recurrent febrile UTIs after re-implantation leading to cystourethroscopy that showed a MRD connected to the prostatic urethra, even though a previous postoperative VCU failed to diagnose the utricle but showed resolution of the VUR. The surgical procedure was the same of the previous patients. UTIs did not occur anymore after surgery, as reported in a one-year follow-up.

\section{Discussion}

PMDS is a disorder of sexual development, which features a failure of involution of Müllerian structures including a uterus, a cervix, fallopian tubes and the upper two thirds of vagina. Their persistency could be influenced by a hormonal imbalance in $\mathrm{AMH}$ and testosterone secretion or the AMH responsiveness of target tissues during a short critical period at the end of the undifferentiated stage (weeks 9 to 10). Undescended testis and posterior forms of hypospadias are often associated as part of the syndrome, due to the above mentioned hormonal imbalance but also secondary to mechanical problem in case of undescended testis. 1,2 The presence of fallopian tubes and their attachment to the testis prevents the testis from descending normally. ${ }^{3}$ EPU is a kind of MDR with a tubular shaped structure communicating with the prostatic urethra. Symptoms such as recurrent UTIs, voiding dysfunctions or urinary incontinence are secondary to urine trapping in the diverticulum. Treatment is aimed at relieving symptoms when present, preserve fertility and prevent neoplastic degeneration. ${ }^{3}$ Our three cases, in fact, were discovered to be affected from an EPU because of symptoms related to urine trapping in the diverticulum. Case $n^{\circ} 1$ came to our attention for pseudo-incontinence; cases $n^{\circ} 2$ and $n^{\circ} 3$ for recurrent UTIs. Removal of the utricle with our approach ended the above-mentioned symptoms. Preservation of fertility is another concern in the management of MDRs since the anatomical proximity to the vas deferens which could be included in the utricle wall or even drain to it. Management of such remnants often require a trade-off between a complete excision of the remnant and minimizing risk to structures vital to fertility. In our 3 cases the vas deferens did not drain into the utricles nor share the same walls. The relationship between PMDS and infertility is still unclear. Men affected from PMDS are often infertile. If, on one hand, infertility could be secondary to an anatomic damage to the reproductive system during a MDR removal or associated malformation repair such as orchiopexy, on the other, could also be part of the underlying genetic disorder that is the base of the persistency of such remnants. It is to consider however that, even if no normal passage from testis to urethra does not exist, in selected patients, testicular sperm extraction is an option. , $^{3,4}$ Further studies are necessary to understand this relation, but in the meanwhile, we believe desirable opting for a surgical approach that could directly view and better respect such delicate structures such as vas deferens. Furthermore, neoplastic degeneration of MDRs has been described. As a matter of fact, Farikullah found 11 reports of Müllerian duct malignancies out of approximately 200 cases over the last 50 -year period. ${ }^{3}$ Current evidence of malignancy arising from MDR is therefore another reason why removal must be taken into consideration seriously by surgeons when dealing with patients with such a pathology and their families. In open surgery, several ways of approach have been advocated to access the retro urethral space and to remove a utricle. All of these procedures are often technically challenging and require prolonged hospitalization. Furthermore, they carry the potential risk of complications, such as infections, incontinence and impotence secondary to injury to the pelvic nerve complex. For these reasons, endoscopic treatment was introduced by many groups with encouraging results but several limitations such as a high recurrence rate. Laparoscopy has become the gold standard treatment in the last two decades since the first intervention described by McDouglas
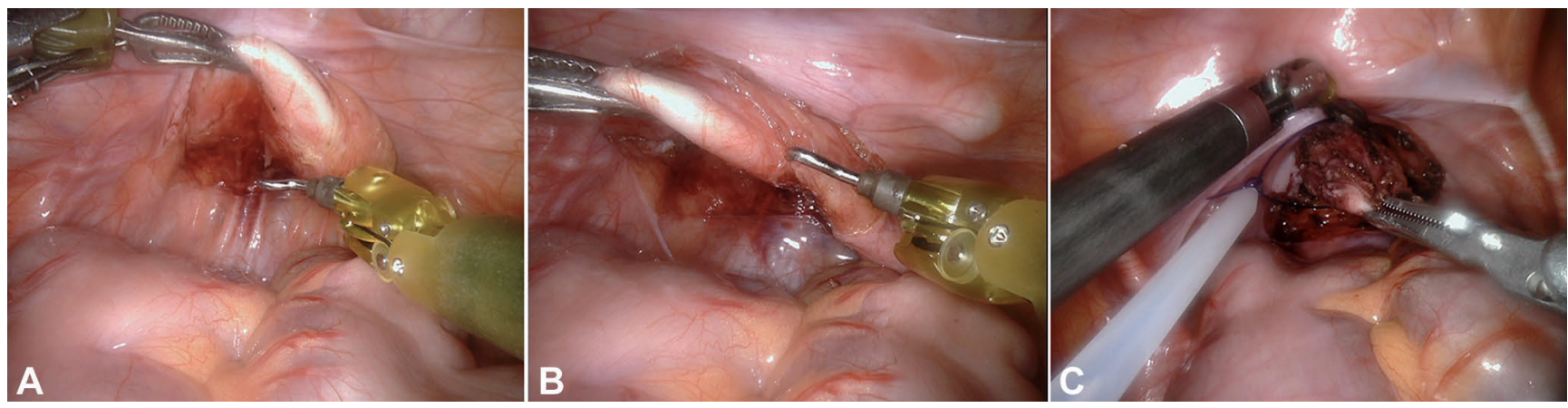

Figure 3. A) The peritoneal reflection covering the dome of the utricle was incised to free the dome with the help of the assistant providing movement of the catheter inside the utricle; B) dissection of the Müllerian duct remnant (MDR) with monopolar hook while the dome is grasped with forceps; C) the MDR neck was secured with two preformed endoscopic loops; D) resection of the utricle just a few millimetres above its junction with the urethra. 
Table 1. Review of the authors performing enlarged prostatic utricle robotic removal in both pediatric and adult age.

\begin{tabular}{|c|c|c|c|c|c|c|}
\hline & $\begin{array}{c}\text { Najamaldin } \\
\text { and Antao, } 2007\end{array}$ & $\begin{array}{l}\text { Hong } \\
\text { et al., } 2011\end{array}$ & $\begin{array}{c}\text { Wu } \\
\text { et al., } 2014\end{array}$ & $\begin{array}{l}\text { Smith-Harrison } \\
\text { et al., } 2015\end{array}$ & $\begin{array}{c}\text { Goruppi } \\
\text { et } a l . ., 2015\end{array}$ & $\begin{array}{l}\text { Lima } \\
\text { et al. }\end{array}$ \\
\hline $\mathrm{N}^{\circ}$ of patients & 1 & 2 & 1 & 1 & 1 & 3 \\
\hline Age & Not available & $29 \mathrm{~m} ; 13 \mathrm{y} 9 \mathrm{~m}$ & $6 \mathrm{~m}$ & $27 y$ & $19 y$ & $7 y ; 7 y ; 3 y$ \\
\hline Follow-up & Not available & $3 \mathrm{~m} ; 4 \mathrm{y} 6 \mathrm{~m}$ & $21 \mathrm{~m}$ & Not available & ly & $1 y ; 1 y ; 1 y$ \\
\hline
\end{tabular}

et al. in 1994. Laparoscopy obviates the above mentioned disadvantages to a large extent because it provides an optimal view, thanks to magnification and permits a fine dissection of MDR with an excellent exposure of all surrounding structures, with minimal trauma to the peritoneal cavity and low incidence of postoperative adhesions. ${ }^{1,2}$ Few cases of robot-assisted excision of retro vesical structures have appeared in literature to date (Table 1).

Najmaldin et al. in their early experience in robotic surgery reported the first case treated with a robot-assisted technology, even though the patient's age was not given. ${ }^{5}$ Hong et al. reported two cases of robotic removal of MDR presenting as symptomatic retro vesical cysts (aged 29 months and 13 years). ${ }^{6}$ In $2014 \mathrm{Wu}$ reported the case of a child affected from PMDS who underwent a successful robot assisted laparoscopic hysterectomy, gonadal biopsy and orchiopexies at the age of 6 months. 7 This was the youngest MRD case reported thus far that was treated with a robotic approach. In reviewed literature, we also found 2 other cases treated with such approach, but in an adult age. ${ }^{4,8}$ Robot-assisted laparoscopy certainly enhances all the advantages of conventional laparoscopy by improving anatomic visualization and surgical precision when performing a challenging dissection within the deep pelvis. We found that the excellent visualization of the retro vesical structures made this technique safe, lowering the risk of injury to the vas deferens, ureters, rectum and bladder neck. It can be considered a valid, safe and effective minimally-invasive technique for the primary treatment of prostatic utricle.

\section{Conclusions}

Removal of persistent Müllerian remnants is aimed at relieving symptoms when present, preserve fertility and prevent neoplastic degeneration. Robot-assisted laparoscopy, by improving anatomic of the retro vesical structures and surgical precision when performing a challenging dissection within the deep pelvis, can be considered a valid, safe and effective minimally-invasive technique for the primary treatment of prostatic utricle.

\section{References}

1. Lima M, Aquino A, Dòmini M, et al. Laparoscopic removal of Müllerian duct remnants in boys. J Urol 2004;171:364-8.

2. Lima M, Aquino A, Dòmini M. Laparoscopic treatment of utricular cyst. In: Bax KNMA, Georgson KE, Rothenberg SS, et al., eds. Endoscopic surgery in infants and children. Berlin: Springer; 2008. pp 737-741.

3. Farikullah J, Ehtisham S, Nappo S, et al. Persistent Müllerian duct syndrome: Lessons learned from managing a series of eight patients over a 10-year period and review of literature regarding malignant risk from the Müllerian remnants. BJU Int 2012;110:E1084-9.

4. Smith-Harrison LI, Patel MS, Smith RP, Schenkman NS. Persistent Müllerian duct structures presenting as hematuria in an adult: Case report of robotic surgical removal and review of the literature. Urol Ann 2015;7:544-6.

5. Najmaldin A, Antao B. Early experience of tele-robotic surgery in children. Int J Med Robot 2007;3:199-202.

6. Hong YK, Onal B, Diamond DA, et al. Robot-assisted laparoscopic excision of symptomatic retrovesical cysts in boys and young adults. J Urol 2011;186:2372-8.

7. Wu JA, Hsieh MH. Robot-assisted laparoscopic hysterectomy, gonadal biopsy, and orchiopexies in an infant with persistent Mullerian duct syndrome. Urol 2014;83:915-7.

8. Goruppi I, Avolio L, Romano P, et al. Robotic-assisted surgery for excision of an enlarged prostatic utricle. Int J Surg Case Rep 2015;10:94-6. 